\title{
Multi-Objective Optimization of Massive MIMO 5G Wireless Networks towards Power Consumption, Uplink and Downlink Exposure
}

\author{
Michel Matalatala ${ }^{1, *(D)}$, Margot Deruyck ${ }^{1}$, Sergei Shikhantsov ${ }^{1}{ }^{(\mathbb{C},}$, Emmeric Tanghe ${ }^{1}$, \\ David Plets ${ }^{1}\left(\mathbb{D}\right.$, Sotirios Goudos ${ }^{2}{ }^{(0}$, Kostas E. Psannis ${ }^{3}{ }^{\circledR}$, Luc Martens ${ }^{1}$ and Wout Joseph ${ }^{1}(\mathbb{C})$ \\ 1 Department of Information Technology, Ghent University/IMEC, Technologiepark-Zwijnaarde 126, \\ B-9052 Ghent, Belgium; margot.deruyck@ugent.be (M.D.); sergei.shikhantsov@ugent.be (S.S.); \\ emmeric.tanghe@ugent.be (E.T.); david.plets@ugent.be (D.P.); luc1.martens@ugent.be (L.M.); \\ wout.joseph@ugent.be (W.J.) \\ 2 Department of Physics, Aristotle University of Thessaloniki, 54124 Thessaloniki, Greece; \\ sgoudo@physics.auth.gr \\ 3 Department of Applied Informatics, School of Information Sciences, University of Macedonia, \\ 54006 Thessaloniki, Greece; kpsannis@uom.edu.gr \\ * Correspondence: michel.matalatala@ugent.be
}

Received: 12 October 2019; Accepted: 14 November 2019; Published: 19 November 2019

Featured Application: The proposed method serves for the network planning of massive Multiple Input Multiple Output (MIMO) based 5G networks. This will be beneficial for the mobile network operators who aim to deploy wireless networks that are cost-effective and electromagnetic (EMF) -aware while providing maximal coverage for the users.

\begin{abstract}
The rapid development of the number of wireless broadband devices requires that the induced uplink exposure be addressed during the design of the future wireless networks, in addition to the downlink exposure due to the transmission of the base stations. In this paper, the positions and power levels of massive MIMO-LTE (Multiple Input Multiple Output-Long Term Evolution) base stations are optimized towards low power consumption, low downlink and uplink electromagnetic exposure and maximal user coverage. A suburban area in Ghent, Belgium has been considered. The results show that the higher the number of BS antenna elements, the fewer number of BSs the massive MIMO network requires. This leads to a decrease of the downlink exposure $(-12 \%$ for the electric field and $-32 \%$ for the downlink dose) and an increase of the uplink exposure ( $+70 \%$ for the uplink dose), whereas both downlink and uplink exposure increase with the number of simultaneous served users $(+174 \%$ for the electric field and $+22 \%$ for the uplink SAR). The optimal massive MIMO network presenting the better trade-off between the power consumption, the total dose and the user coverage has been obtained with 37 64-antenna BSs. Moreover, the level of the downlink electromagnetic exposure (electric field) of the massive MIMO network is 5 times lower than the $4 \mathrm{G}$ reference scenario.
\end{abstract}

Keywords: multi-objective; optimization; massive MIMO; exposure; 5G

\section{Introduction}

With the advent of the fifth generation (5G) of mobile wireless communication, the number of mobile subscriptions is expected to increase by 550 million [1]. The challenge for the mobile operators to contain this traffic is high as they need to design the $5 \mathrm{G}$ networks as optimal as possible in terms of power consumption, electromagnetic (EMF) exposure, and/or cost of deployment. The EMF 
exposure is required to comply with the limitations determined by the International Commission on Non-Ionizing Radiation Protection (ICNIRP) or any other organization such as the Institute of Electrical and Electronics Engineers (IEEE) and the US Federal Communications Commission (FCC), given the proliferation of the broadband wireless devices.

Massive multiple input multiple output (MIMO) is expected to be utilized in the upcoming $5 \mathrm{G}$ cellular network to address the unprecedented high data rate requested by the users [2]. In fact, a massive MIMO 5G BS is equipped with large number of antenna elements that makes it possible to serve many users simultaneously through spatial multiplexing. Moreover, since there are many more antennas than the users, beamforming can be used to focus the signal towards the intended user without interfering with the others. These two properties have the effects of increasing both the spectral and energy efficiency of a massive MIMO 5G network [3].

Recently, the research in the area of massive MIMO has mainly focused on the minimum energy efficiency requirement during the planning process, showing a particular attention to the energy consumption of the BSs deployed within the network [4]. Since the regulations on the electromagnetic field are imposing some strict limitations on the level of BS radiated power the general public is exposed to, the network operators are required to rethink their planning process accounting for the EMF exposure constraints [5]. So far, some studies related to the design of massive MIMO (multiple input multiple output) 5G networks have only addressed the downlink (DL) EMF exposure level resulting from the transmission of the base stations (BS) towards the users. In [6,7], the authors addressed the DL EMF exposure due to an array of 100 antennas $(10 \times 10)$ in the context of $5 \mathrm{G}$. The authors in [8] proposed a statistical approach proving that the realistic EMF compliance boundary due to the DL transmissions of a massive MIMO BS is half-reduced compared to the one obtained with the traditional method. Similarly, $[9,10]$ proposed a theoretical model that recommends to consider the actual maximum transmit power level for a realistic assessment of the DL EMF exposure due to a 5G massive MIMO base station, which is used by the International Electrotechnical Commission (IEC [11]). Since the BS transmit power is split over various directions depending on the locations of the users within the area of interest, the actual transmit power level of the 5G BS was found between $7-22 \%$ of the theoretical maximum power. Furthermore, in [12], the authors investigated a hybrid method combining the ray-tracing (RT) and the finite-difference time-domain (FDTD) to evaluate the DL human exposure due to a single massive MIMO base station in an industrial indoor environment. The RT served for the estimation of the propagation channels, while the FDTD was used for the simulation of the exposure of a human phantom. However, since a deployment of billions of connected objects are expected with the $5 G^{\prime}$ 's use case related to massive machine type communication (mMTC) [13], there is also a need to address the level of EMF exposure, resulting from the transmission the devices towards the BSs as they may have a non-negligible contribution to the global network exposure. This is denoted as uplink (UL) exposure.

In this context, the works of [14-16] proposed a model of the UL exposure and investigated its contribution in the indoor or outdoor wireless network planning. In [17], a global EMF exposure metric denoted " Exposure Index (EI)" is proposed to estimate the EMF exposure caused by a wireless cellular network, accounting for both the transmissions of the users' devices (UL exposure) and the BSs (DL exposure). However, all these researches focused on the previous wireless cellular network technologies ( $2 \mathrm{G}, 3 \mathrm{G}$ and $4 \mathrm{G}$ ) rather than the $5 \mathrm{G}$, which is the aim of our work. The main contribution of this study consists in proposing a simulation-based method to design massive MIMO 5G cellular networks such that the BSs' positions and their respective power levels are optimized while jointly complying with the constraints of lower power consumption, lower DL EMF exposure and lower UL EMF exposure. Furthermore, a realistic assessment of EMF exposure of the network based on the actual maximum power rather than the theoretical maximum power is considered here. To the best of our knowledge, no related works yet investigated a design of massive MIMO network in the context of 5G which simultaneously minimizes the power consumption, the DL EMF exposure and the 
UL EMF exposure, while guaranteeing a good coverage and required capacity to accommodate the user demand.

The remainder of this paper is organized as follows. Section 2 describes the 5G system model considered for this paper; in Section 3 the methodology and the metrics dealing with the optimization objectives are investigated, while Section 4 discusses the results of the simulations based on the different scenarios used before concluding in Section 5.

\section{Massive MIMO 5G Networks}

\subsection{System Model}

A multi-cell massive MIMO network system consisting of L cells, each containing one BS with multiple antenna elements is considered in this study, as shown in Figure 1. This model is similar to the ones proposed in [18-20] in which each BS communicates coherently with the single-antenna users using the time division duplex (TDD) operating mode. This latter is considered to obtain the downlink signaling informations from the uplink pilots thanks to the channel reciprocity property it relies on. These operations take place during the coherence time $\tau_{\mathcal{c}}$ when the propagation channels are almost stable with negligible fluctuations. At the beginning of the TDD process, the users send the uplink pilot sequences consisting of $\tau_{p}$ symbols to the BS which estimates the downlink channels before transmitting the requested downlink data. The estimated channels between the $k$ th user in the $l$ th cell and the $m$ th antenna element of the corresponding BS are referred to as the small-scale fading channels which are assumed independent and identically distributed (i.i.d) Rayleigh fading [21].

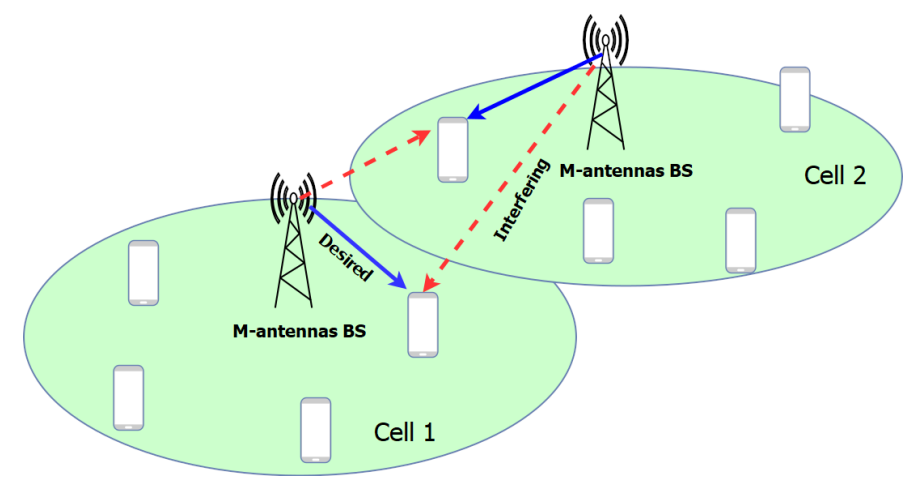

Figure 1. Multi-cell massive Multiple Input Multiple Output (MIMO) network system model.

\subsection{Pilot Contamination}

In a multi-cell environment where all users from different cells simultaneously send the $\tau_{p}$ uplink pilots sequences to their respective BSs, the transmissions of some users could be contaminated (Figure 1) by the transmissions of the other users belonging to the neighboring cells. One way to mitigate this effect is to set apart the cells with the users sharing the same pilot sequences through a reasonable pilot reuse pattern. A pilot reuse factor of 3 [22] has been considered for this study since it provides a better compromise between the lower level of inter-cell interference and the spectral efficiency offered which scales with the number of the uplink pilots [23,24].

Assuming the favorable propagation conditions for the operation of the massive MIMO that make the users' channels directions almost orthogonal when the number of BS antenna elements grow large $[18,25]$, the received signal vector $y_{l k}$ observed at the $k$ th user in the $l$ th cell is modeled as follows [18,26]:

$$
y_{l k}=\underbrace{M \alpha_{M R T} \sqrt{p_{p}} \sqrt{p_{d l k}} \beta_{l k} s_{l k}}_{\text {desired signal }}+\underbrace{M \alpha_{M R T} \sqrt{p_{p}} \sum_{l^{\prime} \neq l}^{L} \sqrt{p_{d l^{\prime} k}} \beta_{l^{\prime} k} s_{l^{\prime} k}}_{\text {contaminated signal }}+\underbrace{n_{d l}}_{\text {noise }}
$$


With $M$ the number of BS antenna elements, $\alpha_{M R T}$ the normalization constant considered to satisfy the transmit power constraint at the BS [26]; $p_{p}$ is the power of the pilots (in W) and $p_{d l k}$ is the DL power of the serving base station (in W) received by the $k$ th user in cell $l ; p_{d l^{\prime} k}$ is the DL power of the interfering BS (in W) in cell $l^{\prime}$ received by the $k$ th user and $n_{d l}$ is the noise level with variance $\sigma_{n}^{2} ; \beta_{l k}$ and $\beta_{l^{\prime} k}$ are the large-scale fadings which are almost constant during the coherence interval and model the effects of the path loss, geometric attenuation and shadowing in cell $l$ and $l^{\prime}$, respectively $[18,27]$.

The Signal to Interference Noise Ratio (SINR) at the receiver side and the sum capacity $C_{l}$ offered by the $l$ th cell can be obtained as follows, respectively $[27,28]$ :

$$
\begin{gathered}
S I N R_{l k}=\frac{M \alpha_{M R T} p_{p} p_{d l k} \beta_{l k}^{2}}{M \alpha_{M R T} p_{p} \sum_{l^{\prime} \neq l}^{L} p_{d l^{\prime} k} \beta_{l^{\prime} k}^{2}+\sigma_{n}^{2}} \\
C_{l}=B \cdot \sum_{k=1}^{K} \gamma^{D L}\left(1-\frac{\tau_{p}}{\tau_{c}}\right) \log _{2}\left(1+S I N R_{l k}\right)(\text { bits } / s)
\end{gathered}
$$

where $\gamma^{D L}$ is the data symbol portions (out of the coherence interval) reserved for DL transmission $\left(\gamma^{D L}=50 \%\right.$ [18]); $B$ is the bandwidth of the channel (in $\mathrm{Hz}$ ) and $K$ is the maximum number of users simultaneously served by the cell.

\section{Method: Massive MIMO Network Design}

In this section, we discuss the different optimization metrics and the algorithm proposed for the design of 5G massive MIMO based network. Furthermore, we describe and formulate the optimization problem investigated while defining the objective fitness functions used, in terms of the power consumption, the downlink exposure and the uplink exposure. Depending on the way the DL and UL EMF exposure are assessed, we consider two types of objective fitness functions:

- A tri-objective fitness function with the downlink exposure and uplink exposure considered as two separate metrics.

- A bi-objective fitness function in which the downlink and uplink exposure are combined into a single exposure metric (the total dose).

\subsection{Problem Description}

In this study, a realistic suburban area of $6.85 \mathrm{~km}^{2}$ in Ghent, Belgium (Figure 2) where a Belgian mobile operator has deployed its $4 \mathrm{G}$ network, has been used for the design of the massive MIMO network. The operator's network consists of 75 possible base stations locations to cover the entire area and provide voice and data services to the users. Two types of optimization problems are considered here, during the design of massive MIMO-LTE networks: maximum area coverage problem and maximum user coverage problem, while simultaneously minimizing the power the consumption, the downlink exposure due to the BSs and the uplink exposure resulting from the transmissions of the users. Here, we use the electric field (in $\mathrm{V} / \mathrm{m}$ ) and the specific absorption rate (SAR in $\mathrm{W} / \mathrm{kg}$ ) as metrics for the downlink and uplink exposure, respectively. These two metrics are standardized by the ICNIRP [29] that defines exposure guidelines for the general public to $61 \mathrm{~V} / \mathrm{m}$ (electric field) and $0.08 \mathrm{~W} / \mathrm{kg}$ (whole-body SAR). Hence, the level of the EMF exposure due to the deployed massive MIMO network should be lower than these limitations to comply with the ICNIRP standards. The details of the problems are presented below. 


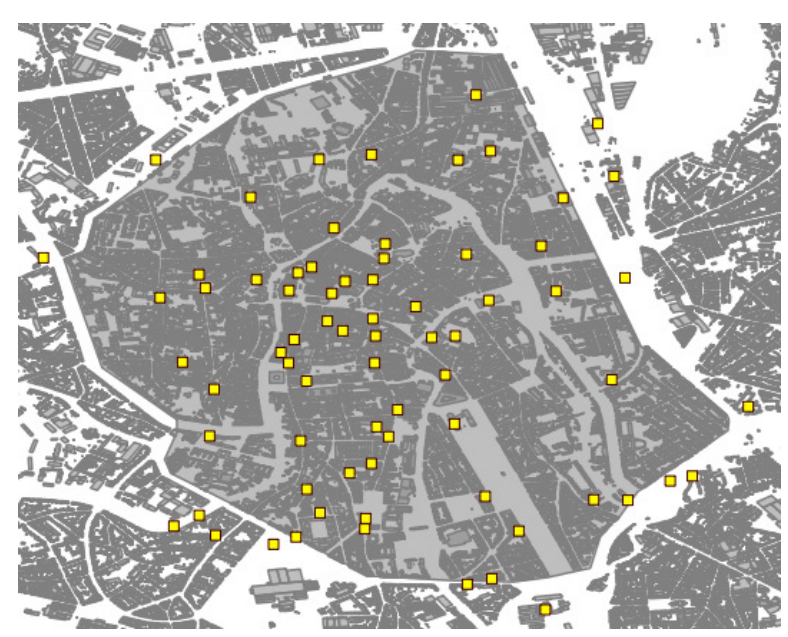

Figure 2. Selected area in Ghent, Belgium and the possible location of the base stations.

We assume that we have a massive MIMO based mobile network that consists of $K$ BSs and $N$ users. The sets of BSs and users are $\mathcal{K}=\{1,2, \cdots, K\}$ and $\mathcal{N}=\{1,2, \cdots, N\}$, respectively. Moreover, we define the set of power values of each BS. This is denoted with $\mathcal{P}=\left\{p_{1}, p_{2}, \cdots, p_{K}\right\}$. We consider the binary variable, $x_{k n}$ that describes the association of the $k$-th BS with the $n$-th user and can be formulated as

$$
x_{k n}= \begin{cases}1, & \text { if } n \text {-th user is associated with the } k \text {-th BS } \\ 0, & \text { otherwise. }\end{cases}
$$

Moreover, we define the binary variable $y_{k}$ that describes the operation or not of the $k$-th BS and can be formulated as

$$
y_{k}= \begin{cases}1, & \text { if BS } \mathrm{k} \text { is turned on } \\ 0, & \text { otherwise. }\end{cases}
$$

Additionally, we can define the discrete variable, $p_{k}$ that describes the transmission power value of the $k$-th BS as $p_{k} \in\left\{0,1,2, \cdots, p_{t}\right\}, \forall k \in \mathcal{K}$. where $p_{t}$ is the maximum allowable transmission power for a BS according to $3 \mathrm{GPP}$ recommendations ( $p_{t}=43 \mathrm{dBm}$ in this work). Thus, we define the solution vector to an integer vector that contains the active or not BSs, the operating power in $\mathrm{dBm}$ and the users associated.

\subsubsection{Tri-Objective Fitness Function}

The tri-objective fitness functions accounting for the power consumption, the downlink exposure and the uplink exposure are defined as follows:

1. Power consumption fitness function

The power consumption fitness function is defined as follows:

$$
f_{1}^{3}=\min _{\{y, p\}}\left(1-\frac{\sum_{k \in \mathcal{K}} P_{\text {calc }}\left(y_{k} p_{k}\right)}{P_{\max }}\right)
$$

where $P_{\text {calc }}()$ is the power consumption of the designed network in $\mathrm{W}$ consisting of the powers consumed by the analog, the baseband and the circuit components [30]; $P_{\max }=K \times P_{\text {calc }}\left(p_{t}\right)$ is the power consumption of the entire network when all BSs are assumed active and transmitting at maximum power $(43 \mathrm{dBm})$. 
2. Downlink exposure fitness function

The below formula accounts for the downlink EMF exposure:

$$
f_{2}^{3}=\min _{\{y, p\}}\left(1-\frac{E_{k \in \mathcal{K}}\left(y_{k} p_{k}\right)}{E_{\max }}\right)
$$

where $E()$ is the electric field in $(\mathrm{V} / \mathrm{m})$ within the designed network-covered area due to the downlink EMF exposure. This latter is modeled similarly to [31,32] in terms of the total EMF exposure $E$ as the weighted average (over the area and the 40 simulations) of the median value $E_{50}$ of the electric field strengths in the area and the 95th percentile $E_{95}$ of the electric field strengths in the same area:

$$
E=\alpha_{1} \cdot E_{50}+\alpha_{2} \cdot E_{95}
$$

$E_{\max }$ is the maximum DL exposure in $[\mathrm{V} / \mathrm{m}]$ obtained when all BSs are assumed active and transmitting at maximum power with the maximum number of antenna elements mounted on each BS. This approach has been adopted since the favorable propagation conditions of the massive MIMO are assumed, in which the individual antenna's propagation channels are mutually uncorrelated. $\alpha_{1}$ and $\alpha_{2}$ are the weighting coefficients related to $E_{50}$ and $E_{95}$ respectively and have been chosen of equal importance $\left(\alpha_{1}=\alpha_{2}=0.5\right)$.

From a practical point of view, with the use of multiple antenna elements, the BS does not always transmit at maximum power since the power is split among different directions depending on the locations of the users within the area of interest [9]. So, in 5G massive MIMO BS, the realistic power level EIRP per antenna element contributing to the EMF exposure is significantly lower compared to the theoretical maximum radiated power $\mathrm{EIRP}_{\text {th }}$ since the duty cycles are applied $\left(\mathrm{EIRP}_{\text {real }}=\mathrm{EIRP}_{\text {th }}[\mathrm{in} \mathrm{W}] * \mathrm{DC}_{\mathrm{TDD}} * \mathrm{DC}_{\mathrm{SPA}}\right)$. Even for very large degrees of system utilization, the authors in [9] proved that the realistic power level can take values between 7-22\%, based on the user distributions considered in both azimuth and elevation. In this analysis, since the users are uniformly distributed in azimuth, the corresponding spatial duty cycle is set to $15 \%$ [9].

To evaluate the electric field created by an antenna element A of a massive MIMO 5G base station, we evaluate the DL exposure at a grid as in [32] with constant distances between two different grid points in both x-and-y axes. For each grid point i, the electric field $E_{i, A}$ due to $\mathrm{A}$ in $5 \mathrm{G}$ is modeled as follows [31,33]:

$$
E_{i, A}=10 \frac{E I R P_{t h}+10 \log _{10}\left(D C_{T D D}^{D L} * D C_{S P A}\right)-43.15+20 \cdot \log _{10}(f)-P L_{d}}{20}
$$

where $f$ is the operating frequency in $\mathrm{MHz} ; P L_{d}$ is the path loss in $\mathrm{dB}$; $\operatorname{EIRP}_{\text {th }}$ is the theoretical maximum radiated power in $\mathrm{dBm} ; \mathrm{DC}_{\mathrm{TDD}}^{\mathrm{DL}}$ is the TDD duty cycle in the downlink $\left(\mathrm{DC}_{\mathrm{TDD}}^{\mathrm{DL}}=0.75\right)$ capturing the fact that the BS does not transmit continuously and $\mathrm{DC}_{\mathrm{SPA}}(=0.15)$ is the spatial duty cycle accounting for the fraction of the BS transmit power towards the direction of the user [9]. The total EMF exposure $\mathrm{E}_{\mathrm{i}}$ at the considered location $i$ (grid point), due to the transmission of all $N$ base stations in the area, each with $M$ antenna elements, is given by the below expression:

$$
E_{i}=\sqrt{\sum_{A=0}^{N \cdot M} E_{i, A}^{2}}
$$

With $E_{i, A}$ is the EMF exposure at a location $i$ due to a single antenna element, calculated via Equation (9).

3. Uplink exposure fitness function 
The UL EMF exposure fitness function is given by the below formula:

$$
f_{3}^{3}=\min _{\{x, y\}}\left(1-\frac{\underset{k \in \mathcal{K}}{S A R_{u l}\left(x_{k n} y_{k}\right)}}{S A R_{\max }}\right)
$$

With $S A R_{u l}()$ is the uplink whole-body specific absorption rate in $(\mathrm{W} / \mathrm{Kg})$ due to the transmissions of the users covered by the designed network. $S A R_{u l}$ is obtained as a weighted average of the median and the 95th percentile values over the 40 simulations and over all users:

$$
S A R_{u l}=\psi_{1} \cdot S A R_{u l 50}+\psi_{2} \cdot S A R_{u l 95}
$$

$S A R_{\text {ulmax }}$ is the uplink whole-body specific absorption rate in $\left(\frac{\mathrm{W}}{\mathrm{Kg}}\right)$ due to the transmission of the users at maximum transmit power $(23 \mathrm{dBm}) . S A R_{\text {ulmax }}$ is obtained similarly to [31] in terms of weighted average as follows:

$$
S A R_{u l \max }=\psi_{1} \cdot S A R_{u l \max 50}+\psi_{2} \cdot S A R_{\text {ulmax } 95}
$$

The whole-body $S A R_{u l}$ in $\left(\frac{\mathrm{W}}{\mathrm{Kg}}\right)$ due to a user's uplink traffic is obtained as per the formula below:

$$
\begin{gathered}
S A R_{u l}=P_{a c t} \cdot D C_{T D D}^{U L} \cdot S A R_{r e f}^{U L} \\
P_{a c t}=10^{\frac{\max (-26, \min (r x s \operatorname{sens}+P L, 23))}{10}}
\end{gathered}
$$

where $D C_{T D D}^{U L}$ is the uplink duty cycle of the TDD network mode of operation. This parameter accounts for the relative transmission time with the TDD mode. Here, we assume a value of 0.25 as proposed in [9]. $S A R_{r e f}^{U L}$ is the uplink reference whole-body specific absorption rate in $\left(\frac{\mathrm{W}}{\mathrm{Kg}}\right)$ for $1 \mathrm{~W}$ of transmitted power $\left(S A R_{r e f}^{U L}=0.0052\left(\frac{\mathrm{W}}{\mathrm{Kg}}\right)\right)$. This value has been obtained by simulations using the numerical tool (Sim4life) with $1 \mathrm{~W}$ of transmitting power, using a dipole at $3.7 \mathrm{GHz}$, positioned next to the right ear. $P_{a c t}$ is the actual transmit power of the user's device in (W). The value of $P_{\text {act }}$ ranges from -26 to $23 \mathrm{dBm}$. $P L$ is the propagation path loss experienced by the user (in $\mathrm{dB}$ ) and rxsens is the sensitivity of the base station (in (dBm)) for maintaining the user's call defined as follows:

$$
\text { rxsens }=T N+N F+S N R
$$

With $\mathrm{NF}$ is the noise figure in $(\mathrm{dB})(\mathrm{NF}=7 \mathrm{~dB}$ [34]), SNR is the signal to noise ratio in $(\mathrm{dB})$ and $\mathrm{TN}$ is the thermal noise in $(\mathrm{dB})$ defined as follows:

$$
T N=10 \cdot \log _{10}\left(K_{B} \cdot T \cdot B\right)
$$

where $\mathrm{K}_{\mathrm{B}}$ is the Boltzman constant $\left(1.38 \times 10^{-23}(\mathrm{~J} / \mathrm{K})\right)$, $\mathrm{T}$ is the reference temperature in Kelvin $(\mathrm{T}=290 \mathrm{~K})$ and $\mathrm{B}$ is the bandwidth.

\subsubsection{Tri-Objective Optimization Problem Formulation}

As the three objectives can scarcely be met simultaneously during the operation of the network, a utility function is built by linearly combining $f_{1}^{3}, f_{2}^{3}$ and $f_{3}^{3}$ to easily solve this tri-objective problem 
and find the possible solutions with the best compromises. Thus, this tri-objective problem can be formulated as follows:

$$
\begin{array}{ll}
\text { P1: } \min _{\{y, x, p\}} & \omega_{1,3} f_{1}^{3}+\omega_{2,3} f_{2}^{3}+\omega_{3,3} f_{3}^{3} \\
\text { s.t. } \quad & C_{1}: y_{k} \in\{0,1\}, \forall k \in \mathcal{K}, \\
& C_{2}: p_{k} \in\left\{0,1,2, \cdots, p_{t}\right\}, \forall k \in \mathcal{K}, \\
& C_{3}: x_{k n} \in\{0,1\}, \forall n \in \mathcal{N}, \forall k \in \mathcal{K}, \\
& C_{4}: \sum_{k=1}^{K} x_{k n}=1, \forall n \in \mathcal{N}, \\
& C_{5}: \sum_{j=1}^{3} \omega_{j, 3}=1, \\
& C_{6}: \frac{\sum_{j=1}^{K} \sum_{i=1}^{N} x_{i j}}{N} \geq 0.95,
\end{array}
$$

where $\omega_{1,3}, \omega_{2,3}, \omega_{3,3}$ are the weights indicating the relative importance (preference) of the objective functions and are determined arbitrary based on the prior knowledge on the priority of either objective. The constraint $C_{1}$ denotes the operation or not of the $k$-th BS. Similarly, the constraint $C_{2}$ denotes the transmission power. The constraint $C_{3}$ denotes the association or not between user $n$ and BS $k$. Moreover, the constraint $C_{4}$ expresses the unique association between one user $n$ with BS $k$ at the same time. Constraint $C_{5}$ denotes that the weights sum is equal to one [35]. Additionally, the constraint $C_{6}$ does not allow the percentage of users served by a BS to be below $95 \%$, as per the radio network planning requirements.

Given that a linear function is convex, the utility function which is a linear combination of convex functions $f_{1}^{3}, f_{2}^{3}$ and $f_{3}^{3}$ is convex as well. Therefore, the problem can be treated as a multi-objective optimization convex problem since the utility or the global fitness function is minimized towards the constraints which are convex as well. The higher the global fitness function, the better the network is optimized as the individual objectives are minimized. We then apply the traditional weighted sum method to resolve the multi-objective optimization problem formulated earlier as the decision maker may have a knowledge of the objective's priority. For each new combination of the weights, the 5G networks are designed by means of system-level simulations using the capacity-based network deployment tool of $[36,37]$ extended with the appropriate optimization algorithm described further in this section. The Pareto optimal concept is then used to compare the different solutions obtained in order to identify the ones with better trade-off between the constraints.

\subsubsection{Bi-Objective Fitness Function}

The bi-objective fitness function accounts for the power consumption and the global EMF exposure. The latter combines the effects of both the downlink and uplink EMF exposure in terms of the total dose, which is the sum of the downlink dose due to the transmission of the BSs and the uplink dose resulting from the user's devices. These fitness functions are defined as follows:

1. Power Consumption fitness function

Here, the fitness function $f_{1}^{\prime}$ that addresses the power consumption objective is similar to Equation (6).

2. Dose (EMF) fitness function

The dose fitness function accounts for the optimization of both the downlink and uplink exposure through a global metric as follows:

$$
f_{2}^{\prime 2}=\min _{\{y, p\}}\left(1-\frac{\underset{k \in \mathcal{K}}{\operatorname{dose}}\left(y_{k} p_{k}\right)}{\text { dose }_{\max }}\right)
$$


With dose ()$\left(\frac{\mathrm{W} \cdot \mathrm{s}}{\mathrm{Kg}}\right)$ the total dose due to the designed network and dose $\mathrm{max}_{\max }\left(\frac{\mathrm{W} \cdot \mathrm{s}}{\mathrm{Kg}}\right)$ the total dose due to the entire network when assuming all the BSs are active and working with the maximum antenna array configuration (256 antenna elements transmitting at max power and all devices active in UL with max power). The dose is defined by the expression below:

$$
\text { dose }=d^{U L}+d^{D L}
$$

where:

(a) $\mathrm{d}^{\mathrm{DL}}\left(\frac{\mathrm{W} \cdot \mathrm{s}}{\mathrm{Kg}}\right)$ is the SAR (whole-body or localized) value for DL multiplied by the time spent in the configuration:

$$
d^{D L}=T_{d}^{D L} \cdot S A R^{D L}
$$

With $\mathrm{T}_{\mathrm{d}}^{\mathrm{DL}}$ the downlink exposure duration in seconds (s) and $S A R^{D L}$ the whole-body or localized $S A R$ induced by the BS in $\left(\frac{\mathrm{W}}{\mathrm{Kg}}\right)$. In this study, we focus on the whole-body $S A R$ :

$$
S A R^{D L}=\sum_{B S_{i}} S_{B S i} \cdot S A R_{r e f}^{D L}
$$

$S A R_{r e f}^{D L}$ is the downlink reference whole-body or localized $S A R$ in $\left(\frac{\mathrm{W}}{\mathrm{Kg}}\right.$ per $\left.\frac{\mathrm{W}}{\mathrm{m}^{2}}\right)$, obtained by means of simulations performed for a horizontal single incident plane wave with an incident power density of $1 \frac{\mathrm{W}}{\mathrm{m}^{2}}$, using numerical electromagnetic simulation software (Sim4life) and $S_{B S i}$ the received power density due to the base station at a location $i$ in $\left(\frac{\mathrm{W}}{\mathrm{m}^{2}}\right)$, obtained as follows:

$$
S_{B S_{i}}=\frac{E_{B S_{i}}^{2}}{120 \cdot \pi}
$$

With $E_{B S_{i}}$ the electric field strength in $[\mathrm{V} / \mathrm{m}]$ due to a base station at a location $i$ obtained as in Equation (10);

(b) $d^{U L}$ is $S A R^{U L}$ (whole-body or localized) values for UL, multiplied by the actual transmit time:

$$
d^{U L}=T_{d}^{U L} \cdot S A R^{U L}
$$

$T_{d}^{U L}$ is here assumed to be $35 s$ within the considered time frame of an hour [16] and $S A R^{U L}$ the uplink whole-body or localized $S A R$ induced by the BS obtained as in Equation (14).

\subsubsection{Bi-Objective Optimization Problem Description}

Similarly to P1, the bi-objective optimization problem can be formulated as follows:

$$
\begin{array}{ll}
\text { P2: } \min _{\{y, x, p\}} & \omega_{1,2} f_{1}^{\prime 2}+\omega_{2,2} f_{2}^{\prime 2} \\
\text { s.t. } \quad & C_{1}: y_{k} \in\{0,1\}, \forall k \in \mathcal{K}, \\
& C_{2}: p_{k} \in\left\{0,1,2, \cdots, p_{t}\right\}, \forall k \in \mathcal{K}, \\
& C_{3}: x_{k n} \in\{0,1\}, \forall n \in \mathcal{N}, \forall k \in \mathcal{K}, \\
& C_{4}: \sum_{k=1}^{K} x_{k n}=1, \forall n \in \mathcal{N}, \\
& C_{5}: \sum_{j=1}^{2} \omega_{j, 2}=1, \\
& C_{6}: \frac{\sum_{j=1}^{K} \sum_{i=1}^{N} x_{i j}}{N} \geq 0.95,
\end{array}
$$

where $C_{1}, C_{2}, C_{3}, C_{4}, C_{5}$ and $C_{6}$ are the same optimization constraints considered in P1; $\omega_{1,2}, \omega_{2,2}$ are the weights indicating the level of priority granted to either objective function. 
Table 1 summarizes the values of the specific parameters considered for the evaluation of the exposure: the reference SARs, the duty cycles and the exposure time duration.

Table 1. Specific parameters considered for the evaluation of the exposure.

\begin{tabular}{lccc}
\hline \multicolumn{1}{c}{ Parameters } & Symbols & Values & Units \\
\hline $\begin{array}{l}\text { Reference Specific } \\
\text { Absorption Rate DL }\end{array}$ & $S A R_{r e f}^{D L}$ & 0.0048 & $\frac{\mathrm{W}}{\mathrm{Kg}}$ per $\frac{\mathrm{W}}{\mathrm{m}^{2}}$ \\
\hline $\begin{array}{l}\text { Reference Specific } \\
\text { Absorption Rate UL }\end{array}$ & $S A R_{r e f}^{U L}$ & 0.0052 & $\frac{\mathrm{W}}{\mathrm{Kg}}$ per W \\
\hline TDD duty cycle in DL & $D C_{T D D}^{D L}$ & 0.75 & {$[-]$} \\
\hline TDD duty cycle in UL & $D C_{T D D}^{U L}$ & 0.25 & {$[-]$} \\
\hline Spatial duty cycle in DL & $D C_{S P A}$ & 0.15 & {$[-]$} \\
\hline Time duration in DL & $T_{d}^{D L}$ & 3600 & $\mathrm{~s}$ \\
\hline Time duration in UL & $T_{d}^{U L}$ & 35 & $\mathrm{~s}$ \\
\hline
\end{tabular}

\subsection{Optimization Algorithm}

In this paper, we proceed with the simulations of the optimal massive MIMO 5G networks to investigate the scenarios described above. We apply a capacity-based network deployment tool proposed in [19], in which the optimization algorithm in Figure 3 is implemented. The optimization algorithm of the tool is based on the BS-user association principle and optimizes the initial set of the base stations deployed in the area of interest while meeting the following optimization constraints: (i) the obtained network must have the least possible power consumption, (ii) the DL and UL EMF exposure resulting from the obtained $5 \mathrm{G}$ networks must be minimal, (iii) the network must respond to the instantaneous bit rates (voice and data) requested by the users and (iv) must provide a coverage for at least $95 \%$ of the users.

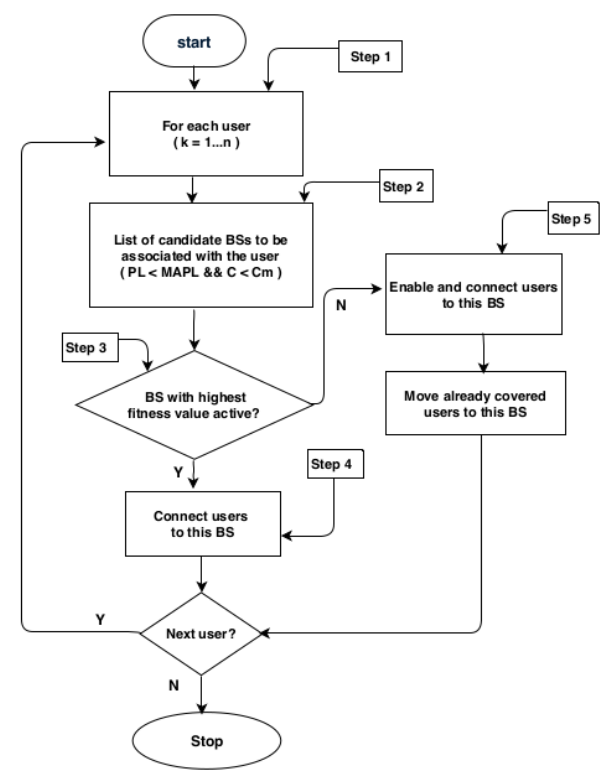

Figure 3. Optimization algorithm implemented in the capacity-based network deployment, designing optimized networks towards power consumption, downlink (DL) and uplink (UL) exposure.

This algorithm is designed in such a way that the decision to associate a user with the base station is related to the value of the fitness function. The algorithm consists of two stages. In the first stage (Figure 3, step 2), a list of possible candidates BSs (active or not) to connect to the users is 
constructed based on the path loss (PL) between the user and each BS. For a BS to be included in the list of candidates BSs, two conditions must be verified:

- For each user, the algorithm evaluates the PL between the user and all the BSs. This PL should be lower than the maximum allowable path loss (MAPL);

- The capacity $C$ of the BS should be high enough to support the high bit rate $C_{u}$ demanded by the user $u\left(\sum_{u} C_{u} \leq C\right)$.

In the second stage (Figure 3, step 3), the algorithm estimates the fitness function for each of the BS inserted in the list of possible candidate BSs. The BS with the highest fitness value is associated with the user and enabled in case the BS was inactive. If there are already enabled (active) BSs within the network, the algorithm checks the possibility to transfer the users already associated with that BS to this newly active BS (Figure 3, step 5). This transfer requires the new enabled BS to present the highest fitness value. If no BS can be enabled, then the user cannot be covered. These operations are repeated for all the users. This results into an optimal massive MIMO 5G network with the highest fitness value. This latter shows a better trade-off between the low-power consumption, the low DL and UL EMF exposure and the users' coverage requirements.

The computation of this algorithm requires the similar input files as in [19]:

- A traffic file containing the maximum number of simultaneous active users and their locations,

- The link budget parameter files (Table 2),

- The 3-dimension (3D) shapes of the environment of study,

- The power consumption file of the individual BS components.

To ensure the massive MIMO requirements in terms of higher data rates imposed by the $5 \mathrm{G}$ applications are met, we consider the 3GPP LTE Rel. 8 path loss model $P L(d)$ (Equation (24)) and the power consumption model proposed in $[19,34]$.

$$
P L(d)=P L_{m e d}(d)+\chi
$$

With $\chi$ a log-normally distributed large-scale fading variable with standard deviation $\sigma$ of $10 \mathrm{~dB}$ [34] and $P L_{\text {med }}(d)$ is the median $P L$ at distance $d$ and defined as follows:

$$
P L_{\text {med }}(d)=40\left(1-0.004 \cdot h_{b}\right) \log _{10}(d)-18 \log _{10}\left(h_{b}\right)+21 \log _{10}(f)+80 \mathrm{~dB}
$$

where $d$ is the distance in $\mathrm{m}$ between the BS and the user; $f$ is the carrier frequency in $\mathrm{MHz}$ and $h_{b}$ is the antenna height of the BS in $\mathrm{m}$.

Table 2. Massive MIMO common link budget parameters.

\begin{tabular}{lc}
\hline \multicolumn{1}{c}{ Parameters } & Values \\
\hline Carrier frequency & $3.7 \mathrm{GHz}^{1}$ \\
Channel bandwidth & $20 \mathrm{MHz}^{1}$ \\
Transmit antenna element gain & $0 \mathrm{dBi}$ \\
Transmit array antenna feed loss & $3 \mathrm{~dB}$ \\
Base Station Total radiated power & $43 \mathrm{dBm}$ \\
Number of MS antenna elements & 1 \\
MS transmit power & $23 \mathrm{dBm}$ \\
Receive antenna element gain & $0 \mathrm{dBi}^{1}$ \\
SNR & $(7.5,15.5,17.2) \mathrm{dB}^{2}$ \\
Implementation loss & $3 \mathrm{~dB}$ \\
RX Noise figure & $7 \mathrm{~dB}$ \\
Other losses (Shadow, fading) & $20 \mathrm{~dB}$ \\
\hline
\end{tabular}

${ }^{1}$ values obtained from [34]. ${ }^{2}$ values of signal-to-noise ratio corresponding to $1 / 2$ BPSK, $1 / 2$ 8PSK, $1 / 2$ 16-QAM modulations, respectively [38]. 


\section{Results}

\subsection{Scenarios}

In this section, we present the different scenarios considered for the design of the massive MIMO network based on the optimization constraints. The operating frequency set to $3.7 \mathrm{GHz}$ (with $20 \mathrm{MHz}$ bandwidth). The results are compared with the reference 4G LTE network operating at 2.6 GHz (with $20 \mathrm{MHz}$ bandwidth) without MIMO. The following scenarios are considered:

- $\quad$ Reference scenario: 4G LTE network operating at 2.6 GHz without MIMO. This is the reference network whose BS positions and power levels are optimized towards power consumption and downlink exposure. It is compared with the designed massive MIMO-LTE networks.

- Suburban information society (300 Mbps for user applications like real-time video gaming) scenarios [34]:

- Scenario 1: the number of users is varied, while assuming all the optimization objectives are of the same importance $\left(\omega_{1,3}=\omega_{2,3}=\omega_{3,3}=0.33\right.$ for the tri-objective optimization problem and $\omega_{1,2}=\omega_{2,2}=0.5$ for the bi-objective one) and the number of BS antenna elements is fixed (256 antenna elements). The number of users varies according to hourly traffic (from a Belgian mobile operator in Ghent), as presented in Figure 4.

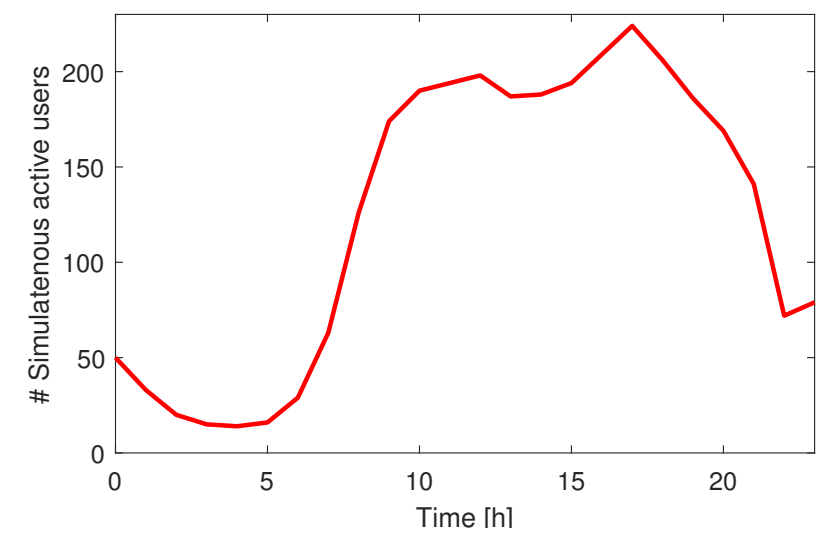

Figure 4. Number of simultaneously served users on a hourly basis in Ghent, Belgium.

- Scenario 2: the number of BS antennas is varied while maintaining the same importance for the optimization objectives $\left(\omega_{1,3}=\omega_{2,3}=\omega_{3,3}=0.33\right.$ for the tri-objective optimization problem and $\omega_{1,2}=\omega_{2,2}=0.5$ for the bi-objective one). Here, the number of simultaneous active users considered for the design is fixed (224 users at busy hour, worst case scenario), while the BSs are equipped with 16, 32, 64, 128 and 256 antenna elements, respectively.

- Scenario 3: The number of simultaneous active users is set to 224 users. Using some sets of combinations $\left(\omega_{1,2}, \omega_{2,2}\right)$, different massive MIMO networks are designed with various BS antenna elements $(16,32,64,128)$, among which only non-dominated Pareto front solutions are retained as optimal ones.

In all these scenarios, the favorable propagation conditions of massive MIMO have been assumed. Moreover, the user equipments (UE) are assumed to have one single transmit antenna. The moderate inter-cell interference resulting from the pilot contamination effects is modeled by the use of the pilot reuse factor $F=3$ [19]. For each considered scenario, we run multiple simulations (40) to ensure we obtain a statistically relevant estimation of the parameters investigated (e.g., the number of BSs, the power consumption and the DL and UL EMF exposure) [32]. 


\subsection{Discussion}

The results obtained by simulations for the different massive MIMO 5G networks are discussed. They are listed in Tables 3 and 4 which refer to scenarios 1 and 2 with respect to the tri-objective and bi-objective optimization problems, respectively. Firstly, the influence of the number of users and BS antenna elements on the massive MIMO network design is assessed, with equally important optimization objectives. Then, in a second step, the optimal solutions jointly meeting the optimization constraints are identified using the Pareto analysis. For each of the considered scenarios, 40 simulations were run to obtain a good estimation of the parameters of interest: the number of BSs, the power consumption, the DL and UL exposure, etc. Only the most important results have been reported within the aforementioned tables in which the 95th-percentile values have been considered for the number of BSs deployed in the network and their corresponding power consumption, while for the exposure (DL and UL), the mean between the 50th-percentile and the 95th-percentile has been used, as in Equation (7).

Table 3. tri-Objective optimization: Impact of the number of antennas elements and the number of users $\left(p_{95}=95\right.$ th percentile $)$.

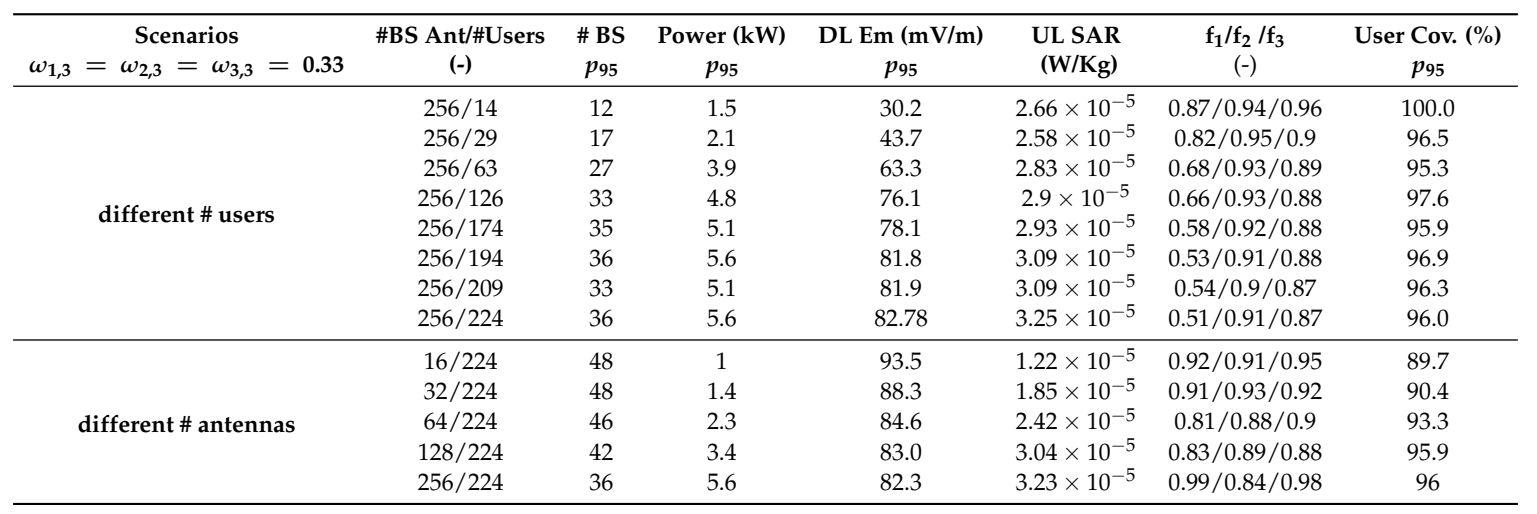

Table 4. bi-Objective optimization: Impact of the number of antennas elements and the number of users ( $p_{95}=95$ th percentile).

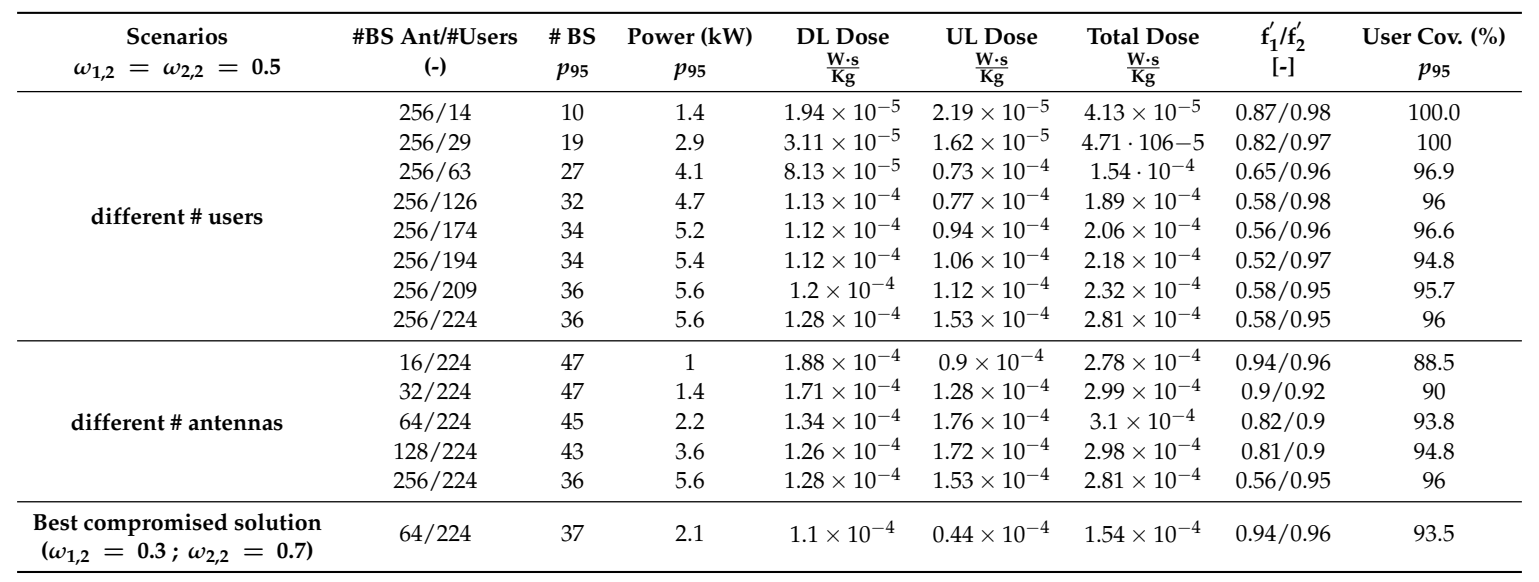

\subsubsection{Impact of the Number of Users on the EMF Exposure}

Figure 5 shows how the electric field $E$ (DL exposure) and the SAR (UL exposure) scale with the number of users, assuming the BSs are equipped with a fixed number of BS antenna elements (256 antenna elements). The increase of the number of users leads to an increase of both $E$ and $S A R$. The $E$ and the SAR increase by $174 \%$ (from $30.2\left(\mathrm{mV} / \mathrm{m}\right.$ ) to $82.8\left(\mathrm{mV} / \mathrm{m}\right.$ )) and $22.2 \%$ (from $2.66 \times 10^{-5}$ to to $3.25 \times 10^{-5}(\mathrm{~W} / \mathrm{Kg})$ ), respectively when the number of users varies from 14 to 224 users (Table 3). 
Similarly, in Figure 6, the DL dose and the UL dose (bi-objective optimization problem) show the same trends, compared to those in Figure 5.

In one side, the UL dose increases ( $+598 \%$, from $2.19 \times 10^{-5}$ with 14 users to $1.53 \times 10^{-4}\left(\frac{\mathrm{W} \cdot \mathrm{s}}{\mathrm{Kg}}\right)$ with 224 users, Table 4) with the number of users before reaching an almost constant value as the optimal number of BSs required to cover the entire area is obtained (Figure 7). On the other side, the DL dose increases by $507 \%$ ( from $1.44 \times 10^{-5}\left(\frac{\mathrm{W} \cdot \mathrm{s}}{\mathrm{Kg}}\right)$ with 14 users to $1.28 \times 10^{-4}\left(\frac{\mathrm{W} \cdot \mathrm{s}}{\mathrm{Kg}}\right)$ with 224 users, Table 4 ). In fact, when the number of users increases within the networks, the number of BSs increases (Figure 7) as well to provide a better coverage to the users spread across the area of interest and cope with the traffic they generate. When the required number of BS is reached, the network coverage keeps improving thanks to the additional beamforming gain provided by the multiple antenna elements the BSs are equipped with. So the users' transmit power slightly increases among the scenarios as soon as a better coverage is achieved with the number of BSs. However, this increase of BSs increases the DL exposure ( $E$ or the DL dose) whose increase rate ( $174 \%$ for $E$ and $560 \%$ for DL dose) is more pronounced than the UL one (22.2\% for UL SAR and 598\% for UL dose).

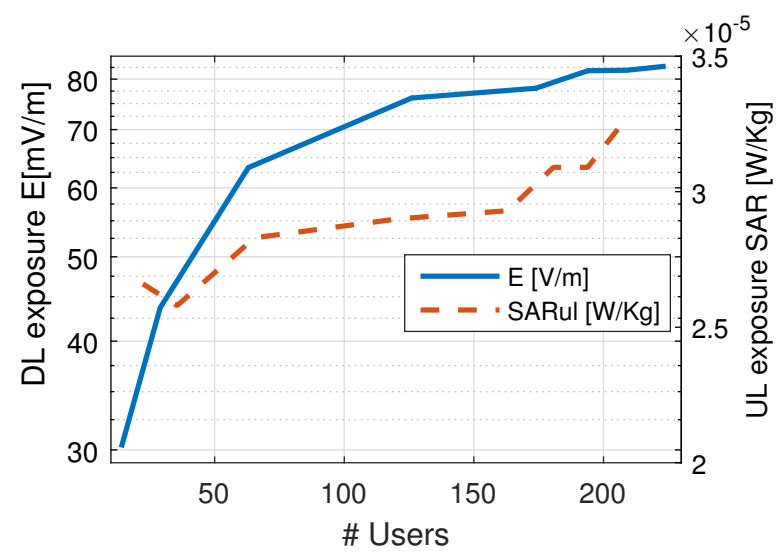

Figure 5. Impact of the number of users on the DL and UL exposure (scenario 1, tri-objective optimization).

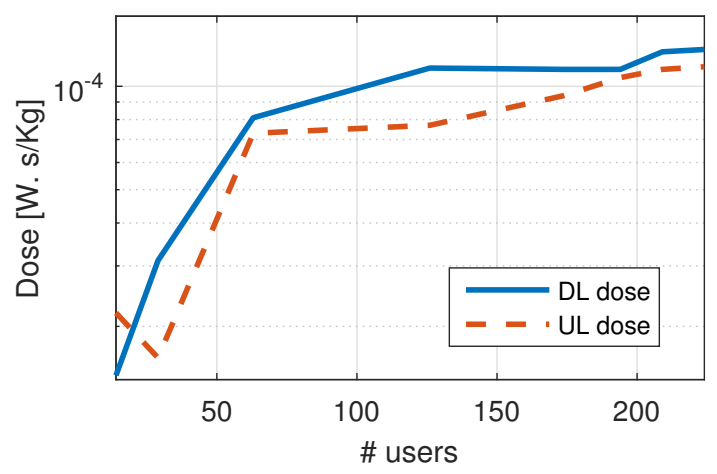

Figure 6. Impact of the number of users on the DL and UL doses (scenario 1, bi-objective optimization).

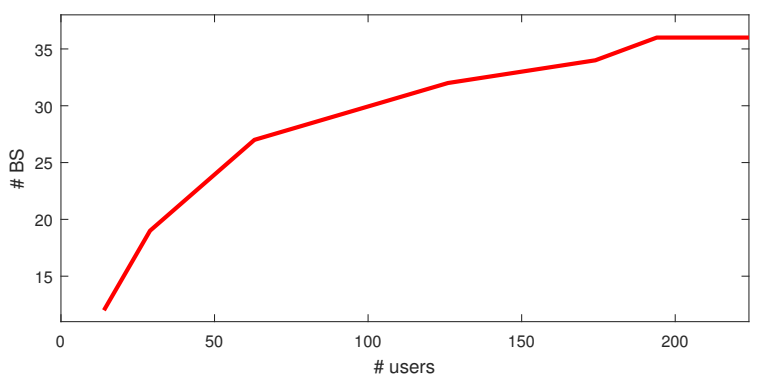

Figure 7. Impact of the number of users on the number of base stations (BSs) deployed. 


\subsubsection{Impact of the Number of Antenna Elements on the EMF Exposure}

Figure 8 shows that when an equal importance is given to the tri-objective optimization problem (power consumption, DL exposure and UL exposure), the increase of the number of BS antenna elements leads to a decrease of the number of BSs deployed in the network (from 48 to 36 BSs, when the antenna elements is increased from 16 to 256, Table 3). This increase of antenna elements at the BS yields to lower power level at each antenna element, as the total radiated power of BS is spread among these multiple antenna elements. Despite this low per-antenna output power level which would have required more BSs with smaller cells' range, the massive MIMO BS benefits from the additional beamforming (BF) gain that helps extend the range of the cells. The BF gain is defined as $10 \cdot \log _{10}(A)$. This results into the deployment of fewer number of BS in the network, which lowers the DL exposure and augments of the UL exposure.

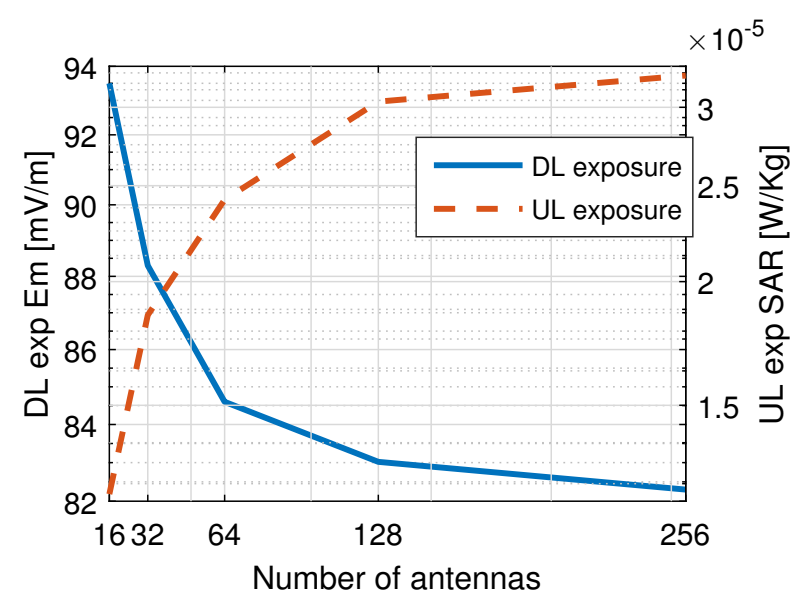

Figure 8. Impact of the number of antenna elements on the DL and UL exposure (scenario 2, tri-objective optimization).

The DL exposure decreases by $12 \%$ (from $93.5(\mathrm{mV} / \mathrm{m})$ with 48 BSs to $82.3(\mathrm{mV} / \mathrm{m})$ with $36 \mathrm{BSs}$ ) when the number of BS antenna elements vary from 16 to 256 respectively (Table 3 ). This is explained by the low transmit power of the antenna elements used at the BS and the fewer BSs needed to provide the coverage to the users (in Table 3, with 256 antenna elements, 25\% less BSs are needed in comparison with the scenario with 16 antenna elements). However, the increase of the UL exposure is related to the fact that the number of BSs deployed in the area of interest decreases with the number of antenna elements until it reaches an optimal value (with 256 antenna elements) requiring the user to transmit with high powers.

The same behavior can be observed when the DL and UL exposure effects are combined into a single metric in terms of dose (Figure 9). The increase of the number of antenna elements leads to a $32 \%$ reduction of the DL dose (from $1.88 \times 10^{-4}$ with 16 antenna elements to $1.28 \times 10^{-4}\left(\frac{\mathrm{W} \cdot \mathrm{s}}{\mathrm{Kg}}\right)$ with 256 antenna elements, Table 4) given the low input power per antenna element. As the coverage improves with the beamforming gain, the more antenna elements the BS is equipped with the fewer number of BSs the network requires to provide a good coverage to the users who will have to adjust their transmit power accordingly to maintain a good radio link with the BS. This results into a $70 \%$ increase of the UL dose: from $0.9 \times 10^{-4}\left(\frac{\mathrm{W} \cdot \mathrm{s}}{\mathrm{Kg}}\right)$ with 16 antenna elements to $1.53 \times 10^{-4}\left(\frac{\mathrm{W} \cdot \mathrm{s}}{\mathrm{Kg}}\right)$ with 256 antennas (see Table 4). 


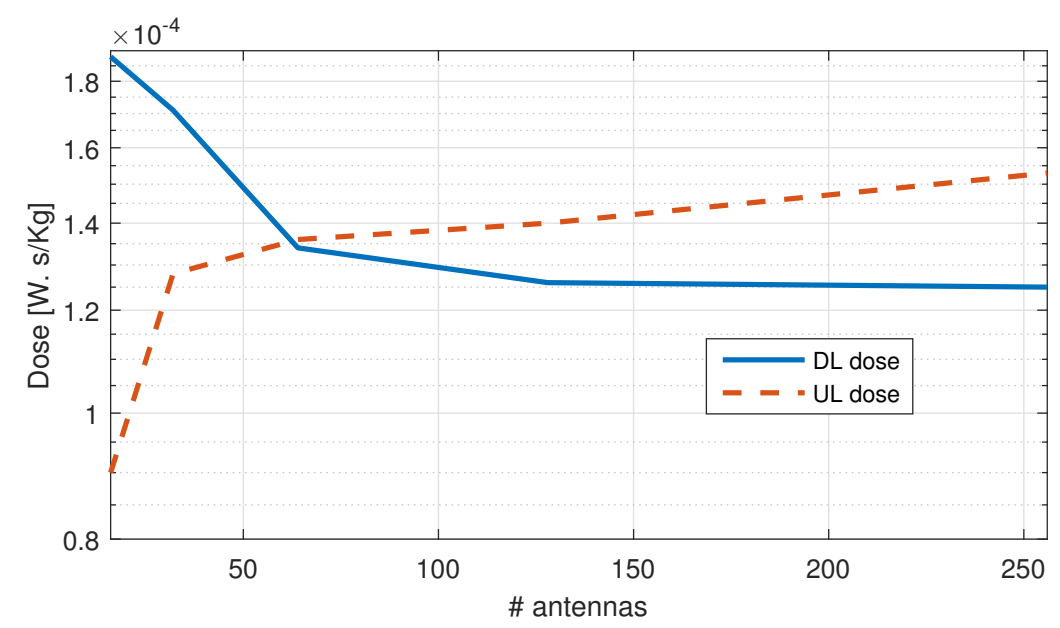

Figure 9. Impact of the number of antenna elements on the DL and UL exposure (scenario 2, bi-objective optimization).

The above results, summarized in Tables 3 and 4, have been obtained with an approach to solve multi-objective optimization problem that assigns the same weight or importance to each objective for a simultaneous optimization in order to assess the influence of both the number of users and BS antenna elements. To this regards, the values of the individual fitness functions $\left(f_{1}, f_{2}\right.$ and $f_{3}$ for the tri-objective optimization and $f_{1}^{\prime}$ and $f_{2}^{\prime}$ for the bi-objective one) have been evaluated and reported on the tables as well. It can be observed that, under the assumption of equal importance of the objective functions, $f_{2}$ and $f_{3}$ are higher compared to $f_{1}$, meaning that the DL exposure (E-field) and the uplink exposure (UL SAR) have a large impact on the optimization since more BSs with low input power are needed to obtain low levels of EMF exposure.

\subsubsection{Pareto Front Analysis}

Here, the scenario 3 is investigated. We focus on the bi-objective optimization problem (P2) for which we would like to estimate the best compromised solution among many others, that satisfy to the low power consumption, low EMF exposure and maximal user's coverage requirements. Given the convexity of the optimization problem, we rely on the Pareto analysis using the weighted sum method. The following combinations of $\omega_{1,2}$ and $\omega_{2,2}$ has been used. First, we vary $\omega_{1,2}$ from 0.1 to 0.9 in steps of 0.1 and derive the corresponding $\omega_{2,2}=1-\omega_{1,2}$. Then, $\omega_{2,2}$ is varied from 0.1 to 0.9 in steps of 0.1 and derive $\omega_{1,2}=1-\omega_{2,2}$. For each combination $\left(\omega_{1,2}, \omega_{2,2}\right)$, we run 40 simulations using the parameters described in scenario 3 and evaluate the power consumption and the total dose due to the network designed. All these points are plotted (Figure10) and the Pareto optimal concept is used to analyze the solution approaches which provide the best compromises between the constraints. Figure 10 shows that the most critical trade-offs are obtained between $1.2 \mathrm{~kW}$ and $2.1 \mathrm{~kW}$, corresponding to a solution with 48 32-antenna BSs $\left(\omega_{1,2}=0.2 ; \omega_{2,2}=0.8\right)$ and a total dose of $1.54 \times 10^{-4}\left(\frac{\mathrm{W} \cdot \mathrm{s}}{\mathrm{Kg}}\right)$ and a solution with 3764 -antenna BSs $\left(\omega_{1,2}=0.3 ; \omega_{2,2}=0.7\right)$ and a total dose of $1.25 \times 10^{-4}\left(\frac{\mathrm{W} \cdot \mathrm{s}}{\mathrm{Kg}}\right)$, respectively. Within that interval, we obtain lower dose and lower power consumption simultaneously with 32 or 64 BS antenna elements. At lower power consumption $(1.2 \mathrm{~kW})$, a $39 \%$ reduction of the total dose is achieved, from $2.56 \times 10^{-4}\left(\frac{\mathrm{W} \cdot \mathrm{s}}{\mathrm{Kg}}\right)$ obtained with a 16-antenna BS $\left(\omega_{1,2}=0.1 ; \omega_{2,2}=0.9\right)$ to $1.54 \times 10^{-4}\left(\frac{\mathrm{W} \cdot \mathrm{s}}{\mathrm{Kg}}\right)$ obtained with a 32-antenna BS. However, the solution with higher power consumption and lower value of total dose (39 BSs, $3.5(\mathrm{~kW})$ and $1.043 \times 10^{-4}\left(\frac{\mathrm{W} \cdot \mathrm{s}}{\mathrm{Kg}}\right)\left(\right.$ with $\omega_{1,2}=0.4$ and $\left.\omega_{2,2}=0.6\right)$ is achieved with BSs equipped with 128 antenna elements and provides better user coverage ( $96 \%$ vs. $94 \%$ ), compared to the most critical trade-offs described above. Since, the objective functions considered here (the power consumption and the total dose) are conflicting, there may be many solutions that satisfy the constraints of low power requirements, low DL and UL EMF exposure. 


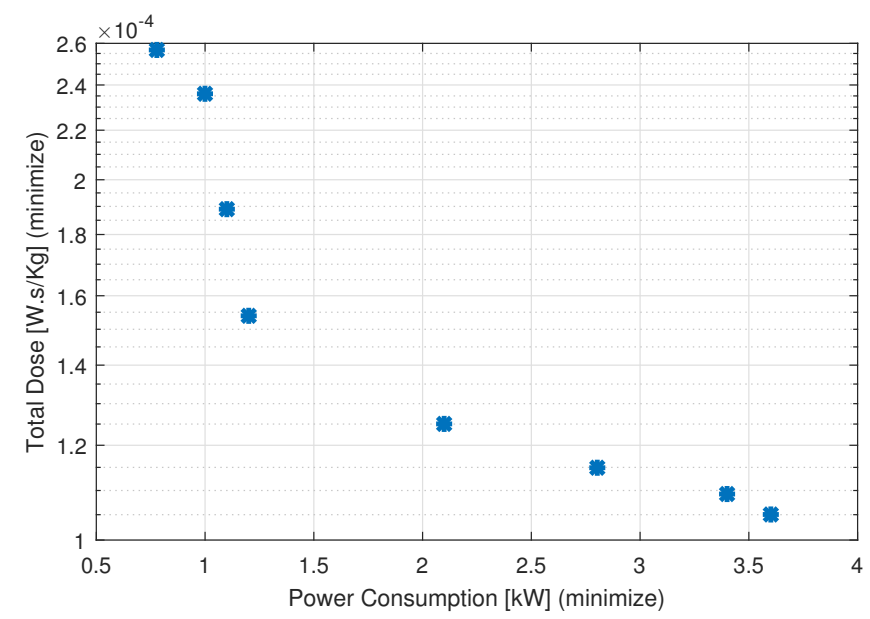

Figure 10. Pareto front for the bi-objective optimization (224 users and various BS antenna elements: $16,32,64,128)$.

\subsubsection{Comparison with 4G LTE Reference Network}

In this section, we compare the levels of EMF exposure due to a 4G LTE BS and a massive MIMO $5 \mathrm{G}$ one in terms of the electric field. A $4 \mathrm{G}$ LTE BS is equipped with a three sector antennas each transmitting at $43 \mathrm{dBm}$, with $10 \mathrm{dBi}$ gain. The massive MIMO 5G BS antennas consist of multiple antenna elements with a total input power of $43 \mathrm{dBm}$. Each antenna element has $0 \mathrm{dBi}$ gain. For a fair comparison, we optimize the power levels of the antennas and the positions of the BSs for both technologies.

The comparison between the cumulative distribution function (CDF) of the 5G downlink EMF exposure in terms of DL electric fields obtained with scenario 2 based on the tri-objective optimization and the CDF of the $4 \mathrm{G}$ reference scenario (optimized for exposure as well) is presented in Figure 11.

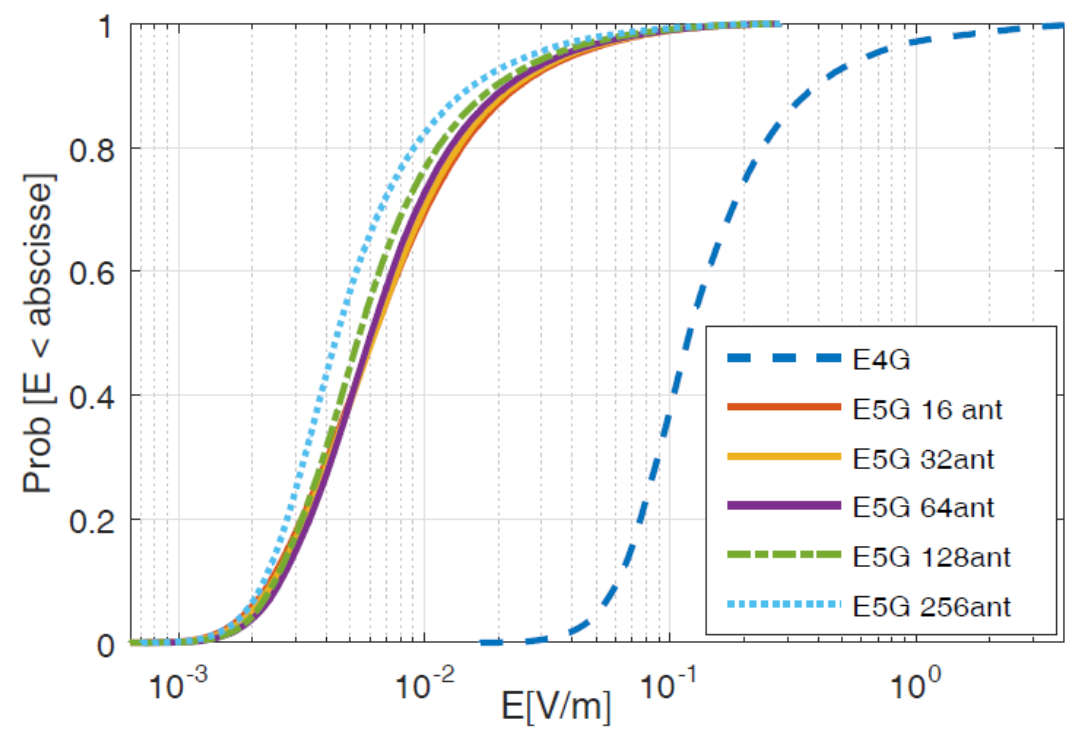

Figure 11. Comparision of the cumulative distribution function (CDF) of the downlink exposure (scenario 2): $4 \mathrm{G}$ vs. $5 \mathrm{G}$ ( $E 4 G$ is the DL electric field due to a $4 \mathrm{G} \mathrm{BS}$, while $E 5 G$ refers to the DL electric field due to a $5 \mathrm{G} B S)$.

The latter shows that the DL EMF exposure level of the massive MIMO 5G network is always lower than the $4 \mathrm{G}$ one at the same probability. The $4 \mathrm{G}$ DL EMF exposure is 5 times higher compared to the DL EMF exposure of the massive MIMO $5 \mathrm{G}$ network $(389.45 \mathrm{mV} / \mathrm{m}$ for the $4 \mathrm{G}$ reference network 
versus $82.8 \mathrm{mV} / \mathrm{m}$ for the $5 \mathrm{G}$ network, Table 3). This is mainly due to the fact that the $4 \mathrm{G}$ BS transmit all the times at maximum optimized power, while in massive MIMO 5G networks, the BS transmit power is lower due to the multiple antennas that are equipped. This reduces the power per antenna element. Moreover, the use of the TDD and spatial duty cycles significantly reduces the BS transmit power. This power reduction at the BS level is compensated by the beamforming gain to help maintain an acceptable radio link with the users. Furthermore, both the $4 \mathrm{G}(389.5 \mathrm{mV} / \mathrm{m})$ and 5G DL $(82.8 \mathrm{mV} / \mathrm{m})$ level of EMF exposure still remains significantly lower than the limits imposed the ICNIRP guidelines (61 V/m [29]). This has been achieved thanks to the control of the power levels and the placements of the BSs.

\section{Conclusions}

In this study, we propose a joint optimization of a multi-cell massive MIMO network towards three objectives: the power consumption, the DL EMF exposure and the UL EMF exposure. Both the DL and UL EMF exposure have been addressed differently. In a first step, the DL and UL EMF exposure are studied separately (E-field for the DL exposure and SAR for the UL exposure), while in a second step, the two objectives are jointly expressed in terms of the dose metric. Assuming the optimization objectives equally important, the results of the simulations show that the DL EMF exposure is more impacted ( $+174 \%$ and $+507 \%$ for $E$ and DL dose, respectively) by the variation of the number of users than the UL EMF exposure ( $+5 \%$ and $+1.3 \%$ for the $S A R$ and UL dose, respectively). Also, for the first time, it has been shown that the increase of the number of BS antennas elements contributes to a $12 \%$ reduction of the DL EMF exposure, at the expense of a higher UL EMF exposure (70\% increase of the UL dose). Furthermore, using Pareto front analysis, we show that the optimal network required to serve 224 users, with a better trade-off of the power consumption and the total dose, is obtained with 37 64-antenna BSs for a power consumption of $2.2 \mathrm{~kW}$ and a total dose of $1.54 \times 10^{-4}\left(\frac{\mathrm{W} \cdot \mathrm{s}}{\mathrm{Kg}}\right)$. Finally, the massive MIMO networks achieve the same performance in terms of user coverage with a DL exposure 5 times lower, compared to the $4 \mathrm{G}$ reference network. Experiments remain the future focus of this research to validate the results in a realistic environment.

Author Contributions: Conceptualization, M.D., E.T., D.P., S.G. and W.J.; methodology, W.J.; software, M.D., M.M.; formal analysis, M.D., D.P., S.G. and W.J.; writing-original draft preparation, M.M.; writing-review and editing, S.S., M.D., E.T., D.P., S.G., K.E.P., L.M. and W.J.; supervision, M.D. and W.J.; project administration, L.M. and W.J.; funding acquisition, W.J.

Funding: This research was funded by the Research Foundation at UGent (BOF-UGent) under the grant agreement number 01W0014.

Acknowledgments: Margot Deruyck is post-doctoral fellow of the FWO-V (Research foundation-Flanders).

Conflicts of Interest: The authors declare no conflict of interest.

\section{References}

1. Gozalvez, J. 5G Worldwide Developments [Mobile Radio]. IEEE Veh. Technol. Mag. 2017, 12, 4-11, [CrossRef]

2. Larsson, E.; Edfors, O.; Tufvesson, F.; Marzetta, T. Massive MIMO for next generation wireless systems. IEEE Commun. Mag. 2014, 52, 186-195, [CrossRef]

3. Björnson, E.; Larsson, E.G.; Debbah, M. Massive MIMO for Maximal Spectral Efficiency: How Many Users and Pilots Should Be Allocated? IEEE Trans. Wirel. Commun. 2016, 15, 1293-1308, [CrossRef]

4. Björnson, E.; Sanguinetti, L.; Hoydis, J.; Debbah, M. Optimal design of energy-efficient multi-user MIMO systems: Is massive MIMO the answer? IEEE Trans. Wirel. Commun. 2015, 14, 3059-3075, [CrossRef]

5. Chiaraviglio, L.; Cacciapuoti, A.S.; Di Martino, G.; Fiore, M.; Montesano, M.; Trucchi, D.; Melazzi, N.B. Planning 5G Networks under EMF constraints: State of the art and vision. IEEE Access 2018, 6, 51021-51037. [CrossRef]

6. Thors, B.; Colombi, D.; Ying, Z.; Bolin, T.; Tornevik, C. Exposure to RF EMF from Array Antennas in 5G Mobile Communication Equipment. IEEE Access 2016, 4, 7469-7478, [CrossRef] 
7. Degirmenci, E.; Thors, B.; Törnevik, C. Assessment of Compliance with RF EMF Exposure Limits: Approximate Methods for Radio Base Station Products Utilizing Array Antennas with Beam-Forming Capabilities. IEEE Trans. Electromagn. Compat. 2016, 58, 1110-1117, [CrossRef]

8. Baracca, P.; Weber, A.; Wild, T.; Grangeat, C. A statistical approach for RF exposure compliance boundary assessment in Massive MIMO systems. In Proceedings of the International Worshop on Smart Antennas (WSA), Bochum, Germany, 14-16 March 2018.

9. Thors, B.; Furuskar, A.; Colombi, D.; Tornevik, C. Time-averaged Realistic Maximum Power Levels for the Assessment of Radio Frequency Exposure for 5G Radio Base Stations using Massive MIMO. IEEE Access 2017, [CrossRef]

10. Methods for the Assessment of Electric, Magnetic and Electromagnetic Fields Associated with Human Exposure; IEC 106; IEC: Geneva, Switzerland, 2018.

11. Determination of RF Field Strength and SAR in the Vicinity of the Radiocommunication Base Stations for the Purpose of Evaluating Human Exposure; IEC 62232; IEC: Geneva, Switzerland, 2011.

12. Shikhantsov, S.; Thielens, A.; Vermeeren, G.; Tanghe, E.; Demeester, P.; Martens, L.; Torfs, G.; Joseph, W. Hybrid Ray-Tracing/FDTD Method for Human Exposure Evaluation of a Massive MIMO Technology in an Industrial Indoor Environment. IEEE Access 2019, 7, 21020-21031, [CrossRef]

13. Dahlman, E.; Mildh, G.; Parkvall, S.; Peisa, J.; Sachs, J.; Selén, Y. 5G radio access. Ericsson Rev. (Engl. Ed.) 2014, 91, 42-47, [CrossRef]

14. Plets, D.; Joseph, W.; Vanhecke, K.; Vermeeren, G.; Aerts, S.; Deruyck, M.; Martens, L. Whole-body and localized SAR and dose prediction tool for indoor wireless network deployments. In Proceedings of the 2014 11th International Symposium on Wireless Communications Systems (ISWCS) 2014, Barcelona, Spain, 26-29 August 2014; pp. 328-332, [CrossRef]

15. Plets, D.; Joseph, W.; Vanhecke, K.; Vermeeren, G.; Wiart, J.; Aerts, S.; Varsier, N.; Martens, L. Joint minimization of uplink and downlink whole-body exposure dose in indoor wireless networks. Biomed Res. Int. 2015, 2015, [CrossRef] [PubMed]

16. Plets, D.; Joseph, W.; Martens, L. Simulation of spatial variations of RF exposure within a macrocell. In Proceedings of the IEEE International Symposium on Antennas and Propagation and National Radio Science Meeting, San Diego, CA, USA, 9-14 July 2017.

17. Varsier, N.; Plets, D.; Corre, Y.; Vermeeren, G.; Joseph, W.; Aerts, S.; Martens, L.; Wiart, J. A novel method to assess human population exposure induced by a wireless cellular network. Bioelectromagnetics 2015, 36, 451-463, [CrossRef] [PubMed]

18. Marzetta, T.; Larsson, E.; Yang, H.; Quoc, H. Fundamentals of Massive MIMO; Cambridge University Press: Cambridge, UK, 2016.

19. Matalatala, M.; Deruyck, M.; Tanghe, E.; Martens, L.; Joseph, W. Optimal Low-Power Design of a Multicell Multiuser Massive MIMO System at $3.7 \mathrm{GHz}$ for $5 \mathrm{G}$ Wireless Networks. Wirel. Commun. Mob. Comput. 2018, 2018, 1-17, [CrossRef]

20. Björnson, E.; Hoydis, J.; Sanguinetti, L. Massive MIMO Fundamentals and State-of-the-Art. 2018. Available online: http://www.commsys.isy.liu.se/ ebjornson/massive_MIMO_WCNC18.pdf (accessed on 12 October 2019).

21. Akbar, N.; Yang, N.; Sadeghi, P.; Kennedy, R.A. Multi-Cell Multiuser Massive MIMO Networks: User Capacity Analysis and Pilot Design. IEEE Trans. Commun. 2016, 64, 5064-5077, [CrossRef]

22. Chataut, R.; Akl, R. Optimal pilot reuse factor based on user environments in 5G Massive MIMO. In Proceedings of the 2018 IEEE 8th Annual Computing and Communication Workshop and Conference (CCWC), Las Vegas, NV, USA, 8-10 January 2018; pp. 845-851, [CrossRef]

23. Bjornson, E.; Larsson, E.G.; Debbah, M. Optimizing multi-cell massive MIMO for spectral efficiency: How Many users should be scheduled? In Proceedings of the 2014 IEEE Global Conference on Signal and Information Processing (GlobalSIP), Atlanta, GA, USA, 3-5 December 2014; pp. 612-616, [CrossRef]

24. Araujo, D.; Maksymyuk, T.; de Almeida, A.; Maciel, T.; Mota, J.; Jo, M. Massive MIMO: Survey and future research topics. IET Commun. 2016, 10, 1938-1946. [CrossRef]

25. Bjornson, E.; Hoydis, J.; Sanguinetti, L. Massive MIMO Networks: Spectral, Energy, and Hardware Efficiency. Found. Trends Signal Process. 2017, 11, 154-655. [CrossRef] 
26. Ngo, H.; Larsson, E.; Marzetta, T. Massive mu-mimo downlink TDD systems with linear precoding and downlink pilots. In Proceedings of the 2013 51st Annual Allerton Conference on Communication, Control and Computing, Monticello, IL, USA, 2-4 October 2013; pp. 293-298.

27. Jabbar, S.; Li, Y. Analysis and Evaluation of Performance Gains and Tradeoffs for Massive MIMO Systems. Appl. Sci. 2016, 6, 268, [CrossRef]

28. Van Chien, T.; Björnson, E.; Larsson, E.G. Joint Power Allocation and User Association Optimization for Massive MIMO Systems. IEEE Trans. Wirel. Commun. 2016, 15, 6384-6399, [CrossRef]

29. Limiting, F.O.R.; To, E.; Fields, M. Icnirp Guidelines for Limiting Exposure To Time - Varying Guidelines for Limiting Exposure To Time-Varying. Health Phys. 1998, 74, 494-522, [CrossRef]

30. Desset, C.; Debaillie, B.; Louagie, F. Modeling the hardware power consumption of large scale antenna systems. In Proceedings of the 2014 IEEE Online Conf. Green Commun. OnlineGreenComm 2014, Tucson, AZ, USA, 12-14 November 2014; pp. 1-6, [CrossRef]

31. Plets, D.; Wout, J.; Vanhecke, K.; Martens, L. Exposure Optimization in Indoor Wireless. Prog. Electromagn. Res. 2013, 139, 445-478. [CrossRef]

32. Deruyck, M.; Tanghe, E.; Plets, D.; Martens, L.; Joseph, W. Optimizing LTE wireless access networks towards power consumption and electromagnetic exposure of human beings. Comput. Netw. 2016, 94, 29-40, [CrossRef]

33. Guidance for Assessment, Evaluation and Monitoring of Human Exposure to Radio Frequency Electromagnetic Fields; ITU-T-REC-K.91; ITU: Geneva, Switzerland, 2012.

34. MAMMOET. Massive MIMO for Efficient Transmission: Deliverables 1.1, Systems Scenarios and Requirements Specifications; MAMMOET: Utrecht, The Netherlands, 2014.

35. Ngatchou, P.; Zarei, A.; El-Sharkawi, A. Pareto Multi Objective Optimization. In Proceedings of the 13th International Conference on Intelligent Systems Application to Power Systems, Arlington, VA, USA, 6-10 November 2005; pp. 84-91, [CrossRef]

36. Matalatala, M.; Deruyck, M.; Tanghe, E.; Martens, L.; Joseph, W. Performance evaluation of 5G millimeter-wave cellular access networks using a capacity-based network deployment tool. Mob. Inf. Syst. 2017, 2017, 3406074, [CrossRef]

37. Deryuck, M.; Joseph, W.; Tanghe, E.; Martens, L. Reducing the power consumption in LTE-Advanced wireless access networks by a capacity based deployment tool. Radio Sci. 2014, 49, 777-787. [CrossRef]

38. Fixed Radio Systems; Parameters Affecting the Signal-to-Noise Ration (SNR) and the Receiver Signal Level (RSL) Threshold in Point-to-Point Receivers; ETSI TR 103 053; ETSI: Sophia Antipolis, France, 2014; pp. 1-22.

(c) 2019 by the authors. Licensee MDPI, Basel, Switzerland. This article is an open access article distributed under the terms and conditions of the Creative Commons Attribution (CC BY) license (http://creativecommons.org/licenses/by/4.0/). 